\title{
Stent-Mediated Gene Delivery for Site-Specific Transgene Administration to the Airway Epithelium and Management of Tracheobronchial Tumors
}

\author{
Robert J. Kruklitis ${ }^{c}$ Ilia Fishbein ${ }^{b}$ Sunil Singhal ${ }^{a}$ Veena Kapoor ${ }^{a}$ Robert J. Levy ${ }^{b}$ \\ Daniel H. Sterman ${ }^{a}$ \\ aSection of Interventional Pulmonology and Thoracic Oncology, Pulmonary, Allergy and Critical Care Division, \\ University of Pennsylvania Medical Center, and ' ${ }^{b}$ Division of Pediatric Cardiology, Children's Hospital of Philadelphia, \\ Philadelphia, Pa., and ' Division of Pulmonary and Critical Care, Lehigh Valley Health Network, Allentown, Pa., USA
}

\section{Key Words}

Non-small cell lung cancer · Tracheobronchial tumors ·

Intra-arterial stents $\cdot \beta$-Galactosidase

\begin{abstract}
Background: Gene therapy is currently under investigation as a means of managing a variety of pulmonary diseases. Unfortunately, gene transfer to bronchial epithelium has been hampered by the lack of stable and efficient transduction. Recent studies have shown that gene vectors could be tethered to the metallic surfaces of intra-arterial stents. This approach enables efficacious and site-specific adenoviral gene delivery to the vascular endothelium. $\mathbf{O b}$ jectives: We hypothesized that airway mesh stents impregnated with viral gene vectors could be used for local gene delivery to benign and malignant bronchial epithelium. Methods: Serotype 5 adenoviral vectors (Ad5, E1-/E3-) containing the reporter genes green fluorescent protein (Ad.GFP) or $\beta$-galactoside/LacZ (Ad.LacZ), or a therapeutic gene, Ad.INF- $\beta$, were coupled to either metallic mesh disks or stents via anti-Ad knob antibodies. These platforms
\end{abstract}

\section{KARGER}

E-Mail karger@karger.com

www.karger.com/res were assessed for their ability to transfect bronchial epithelial cells from both rats and humans, as well as murine (L1C2) and human (A549) lung cancer cell lines. Gene transfer was quantified by fluorescent microscopy, scanning fluorimetry for Ad.GFP, and light microscopy studies assessing $\beta$-galactosidase staining for Ad.LacZ. Metallic mesh and stent-mediated gene transfer was also performed in a murine flank tumor model and in a rat endotracheal tumor model in order to evaluate the therapeutic potential. $\boldsymbol{R e}$ sults: In these studies, murine and human non-small cell lung cancer (NSCLC) cells were successfully transfected with reporter genes in vitro. Ad.LacZ-complexed mesh successfully transfected reporter genes into established murine flank NSCLC tumors. In addition, Ad.LacZ-tethered stents could effectively transfect both tracheobronchial epithelium and submucosal glands in rats. Similar epithelial transfection was achieved in ex vivo human bronchial epithelium. Pilot in vivo experimentation provided data

R.J.K. and I.F. contributed equally to the conduct of this research and the production of this manuscript. 
supporting the concept that therapeutic genes could also be delivered with this technology. In additional pilot in vivo experiments, the growth of murine flank tumors was inhibited by placement of mesh disks coupled with Ad. mulNF- $\beta$, and rats bearing endotracheal tumors demonstrated a trend towards prolonged survival with insertion of Ad.ratINF- $\beta$-tethered stents. Conclusions: Stent-mediated gene delivery successfully enabled site-specific vector administration to target rat and human airway cells in cell culture, organ culture and in vivo. Local tracheobronchial gene delivery via stents could provide a viable clinical solution for overcoming the difficulties encountered with vector delivery within the lungs, in particular by lowering requisite vector titers and by directing desired vectors to areas of interest. This strategy may prove valuable for treating tumors involving the tracheobronchial tree, as well as other nonmalignant tracheobronchial disorders.

(c) 2014 S. Karger AG, Basel

\section{Introduction}

Non-small cell lung cancer (NSCLC) is the most common cause of cancer-related mortality in developed countries. In the USA alone, approximately 170,000 people are diagnosed annually with NSCLC and a near equal amount die from this cause [1]. Early-stage NSCLC can be cured with surgical resection; however, most patients present with advanced-stage disease at diagnosis or recur after initial curative surgery [2]. For these patients, palliative chemotherapy and radiation therapy have demonstrated a minimal improvement in survival $[3,4]$. Therefore, there is a very strong impetus to develop novel therapies to treat this devastating disease.

Gene therapy is a novel modality to treat a wide spectrum of human diseases, and it has progressed considerably in the past several decades [5]. The technological advances of gene transfer have been studied for the treatment of NSCLC [6], as well as a variety of nonmalignant pulmonary disorders, such as cystic fibrosis (CF) and a1antitrypsin deficiency [5]. The flexible bronchoscope, because of its unique access to both large and small airways, serves as an ideal instrument to deliver therapeutic genes to the tracheobronchial tree. Currently, there are three primary mechanisms for delivering gene transfer vectors via the bronchoscope: (1) direct instillation of soluble vector (bronchial, bronchoalveolar lavage); (2) aerosolization of vector via specially designed spray catheters, and (3) direct injection of vector via bronchoscopic needle [7].

Stent-Mediated Gene Delivery
Soluble gene transfer vectors can be delivered through the working channel of a flexible bronchoscope via direct instillation in a manner analogous to bronchial lavage, facilitating focal gene delivery into a specific lobe or segment. After bronchoscopic instillation, however, viral vectors preferentially distribute into the distal airways and, therefore, may be best utilized in the treatment of diffuse parenchymal lung diseases or widespread pulmonary adenocarcinoma $[8,9]$.

For diseases of the respiratory epithelium, such as CF or asthma, the more proximal conducting airways are the targeted site and, therefore, direct bronchoscopic instillation is not the ideal delivery method. Transbronchoscopic sprayer devices have been developed with the goal of achieving more efficient delivery of viral vectors to the conducting airways [10]. These sprayers, which are introduced through the working channel of a flexible bronchoscope, aerosolize the soluble vector into relatively large particles $(10-25 \mu \mathrm{m})$. Solution particles of this size preferentially deposit vectors to the large conducting airways, thus minimizing distal delivery [11]. Although vector aerosolization via nebulizers may offer a simple, noninvasive means of gene delivery to the lung parenchyma, this process may adversely affect vector viability $[7,12]$.

Unfortunately, intrapulmonary gene transfer via bronchoscopic instillation of soluble vector has been quite inefficient, likely due to the innate defense mechanisms of the tracheobronchial mucosa $[7,13]$. Direct bronchoscopic needle injection of therapeutic genes is one reasonable approach for the treatment of accessible bronchogenic malignancies with the advantage of bypassing airway mucosal defense mechanisms. Unfortunately, larger endobronchial tumors may require multiple vector injections, increasing the risk of systemic dissemination. In addition, direct intratumoral gene delivery is not optimal due to rapid clearance of delivered genes from the target tissues.

Previous studies have demonstrated that exposing the surfaces of stainless steel endovascular stents to an aqueous solution of polyallylamine bisphosphonate (PAA$\mathrm{BP})$ results in their chemical activation, thereby enabling the attachment of viral vector-binding agents for local gene delivery. In addition, members of our team previously demonstrated that this approach could be utilized to deliver therapeutic genes to vascular endothelium for prevention of in-stent restenosis [14]. In this study, our goal was to assess whether similar methods of gene delivery would prove successful in benign and malignant tracheobronchial epithelium. 


\section{Material and Methods}

\section{Reagents}

PAA-BP was synthesized for these studies by reacting vinyl bisphosphonate with polyallylamine as previously described [14]. An anti-knob antibody specific for the adenovirus knob fiber protein was obtained from Selective Genetics (San Diego, Calif., USA). All other reagents used were obtained from Sigma-Aldrich (St. Louis, Mo., USA) as ACS reagent-grade compounds.

\section{Coupling of Metallic Mesh Disks or Stents with Cy3-Labeled} Adenoviral Vectors and Estimation of Linkage Efficiency

Stainless steel mesh disks were reversibly tethered with either adenoviral vectors containing the green fluorescent protein (Ad. GFP) gene or the bacterial marker gene, LacZ (Ad.LacZ), as previously described [14]. Briefly, metallic surfaces were exposed to an aqueous solution of PAA-BP as described elsewhere, with subsequent exhaustive rinse in distilled water [14]. This step was followed by sulfhydryl-mediated attachment of a mouse anti-knob antibody using SPDP. The metallic mesh disks were then incubated in a saturating solution of $1 \times 10^{10}$ particles $/ \mathrm{ml}\left(1 \times 10^{9} \mathrm{pfu} /\right.$ $\mathrm{ml}$ ) of adenoviral vector. The efficacy and uniformity of steel surface derivatization with anti-knob antibody was assessed by immunofluorescence using an FITC-labeled secondary anti-mouse antibody (Vector Laboratories, Burlingame, Calif., USA). Additionally, Ad disposition on the mesh surface was visualized employing Cy3-labeled adenoviral vector using the methodology previous published by our group [14]. The surface density of immobilized adenoviruses $\left(\sim 2 \times 10^{9} / \mathrm{cm}^{2}\right)$ was determined previously by the depletion assay as the difference between the Cy3 fluorescent signal $(540 / 580 \mathrm{~nm})$ elicited from nondepleted and metal sample-depleted virus suspensions normalized by the specific fluorescence of labeled adenovirus [14]. This immobilization density corresponds to $1.5 \times 10^{8}$ vector particles $\left(1.5 \times 10^{7} \mathrm{pfu}\right)$ per mesh.

Transduction of Tumor Cell Lines in vitro Using Mesh Metallic Disks with Tethered Gene Vectors

Two NSCLC cell lines, either derived from human (A549 obtained from ATCC) or mouse (L1C2 obtained from Steven Dubinett, MD, University of California, Los Angeles), were used for in vitro mesh experiments. Cells were maintained in DMEM medium with $10 \%$ fetal bovine serum, 100 units $/ \mathrm{ml}$ penicillin $\mathrm{G}, 100 \mu \mathrm{g} / \mathrm{ml}$ streptomycin and $2 \mathrm{~mm}$ glutamine. The Cy3Ad.GFP-tethered mesh disks were placed directly onto the cell monolayer in 24-well plates. The equivalent amounts of free-soluble Cy3Ad.GFP $(1.5 \times$ $10^{8}$ particles) were added to different wells on the same plates. The cultures were examined by fluorescence microscopy (Leica DC 500) and by well-scan fluorimetry (Gemini Max EM) for the intensity and spatial distribution of GFP signal $20 \mathrm{~h}$ following the initiation of transduction.

Transduction of Murine Flank Tumors with Mesh-Mediated

Delivery of Adenoviral Vector

L1C2 tumor cells, grown in vitro, were trypsinized during their logarithmic growth phase, resuspended to $5 \times 10^{5}$ cells $/ 100 \mu \mathrm{l}$ in DMEM without FBS and injected subcutaneously into the right flank of syngeneic Balb/C mice in order to establish flank tumors. When the average tumor volume achieved approximately 125-250 $\mathrm{mm}^{3}$, metallic mesh disks, tethered with either Ad.LacZ or
Ad.INF- $\beta$, were surgically implanted underneath the abdominal musculature in direct contact with the flank tumor $[15,16]$. This procedure was performed using standard surgical techniques under sterile conditions after mice had been anesthetized using a mixture of xylocaine and ketamine administered intraperitoneally. Tumor growth was assessed serially over time using standard calipers. Additionally, in order to assess tumor cell transduction, mice were sacrificed $48 \mathrm{~h}$ after mesh disk implantation and the flank tumors were resected. Adherent mesh disks were removed and the tumors wereflash frozen, sectioned and stained with $\beta$-galactosidase $(\beta$-gal). For comparison, established flank tumors were treated with the usual manner of adenoviral vector delivery - intratumoral injection with Ad.LacZ. A 100- $\mu$ l solution containing $1 \times 10^{10}$ particles $/ \mathrm{ml}$ of Ad.LacZ (total vector amount $1 \times 10^{9}$ viral particles) was directly injected into $\sim 250 \mathrm{~mm}^{3}$ flank tumors. These mice were also sacrificed at $48 \mathrm{~h}$ and the tumors were resected, sectioned and stained with $\beta$-gal. Visual comparison of staining was made by light microscopy.

\section{In vivo Rat Model of Endobronchial Stent-Mediated Marker Gene Delivery}

Fisher 344 rats were anesthetized with xylocaine and ketamine mixture and placed in the supine position. Using standard surgical techniques, a 1.0 - to $1.5-\mathrm{cm}$ incision was made through the skin and the trachea was exposed after dissection of the subcutaneous tissues. A 3-0 silk suture was placed behind the trachea to give counter tension during stent deployment. A small transverse incision was made between two tracheal rings approximately 5-8 rings below the larynx. An 8-mm stainless steel endovascular stent (Boston Scientific Corporation, Natick, Mass., USA) tethered with Ad.LacZ was loaded onto a balloon deployment catheter that was advanced through the incision into the mid-trachea. The balloon was inflated to 8 atm for $15 \mathrm{~s}$ to facilitate stent deployment. Following removal of the deployment catheter, the trachea was closed with two interrupted 5-0 silk sutures.

\section{In vivo Model of Endobronchial Stent-Mediated Therapeutic}

Gene Delivery in Tumor-Bearing Rats

A similar technique for rat tracheal stent placement was utilized in a pilot experiment involving the insertion of stainless steel stents coupled with therapeutic transgenes. A rat model of tracheal tumor was created by injection of $2 \times 10^{5}$ cells of the II- 45 mesothelioma cell line syngeneic for the Fisher 344 rat [17]. As demonstrated in figure 5, local injection of these cells resulted in tumor growth within the trachea similar to that of human tumors, with invasion beyond the tracheal cartilages and airway obstruction resulting in death in the preponderance of animals. We were able to insert uncoupled stainless steel stents through the transtracheal approach described above in tumor-bearing animals. In addition, we tethered a recombinant adenovirus carrying the rat IFN- $\beta$ gene (Ad.ratIFN- $\beta$; BiogenIDEC, San Diego, Calif., USA) to stainless steel stents ( $\sim 10^{9}$ physical viral particles per stent), and then inserted these 'therapeutic' stents transtracheally into tumor-bearing animals (fig. 5).

\section{Ex vivo Gene Delivery to Human Bronchial Epithelium}

Distal fifth-to-seventh generation human airways with an internal diameter of approximately 6-10 $\mathrm{mm}$ were obtained from surgical specimens by sterile dissection from surrounding lung tissue. The explanted bronchus was placed into DMEM with 10\% FBS 


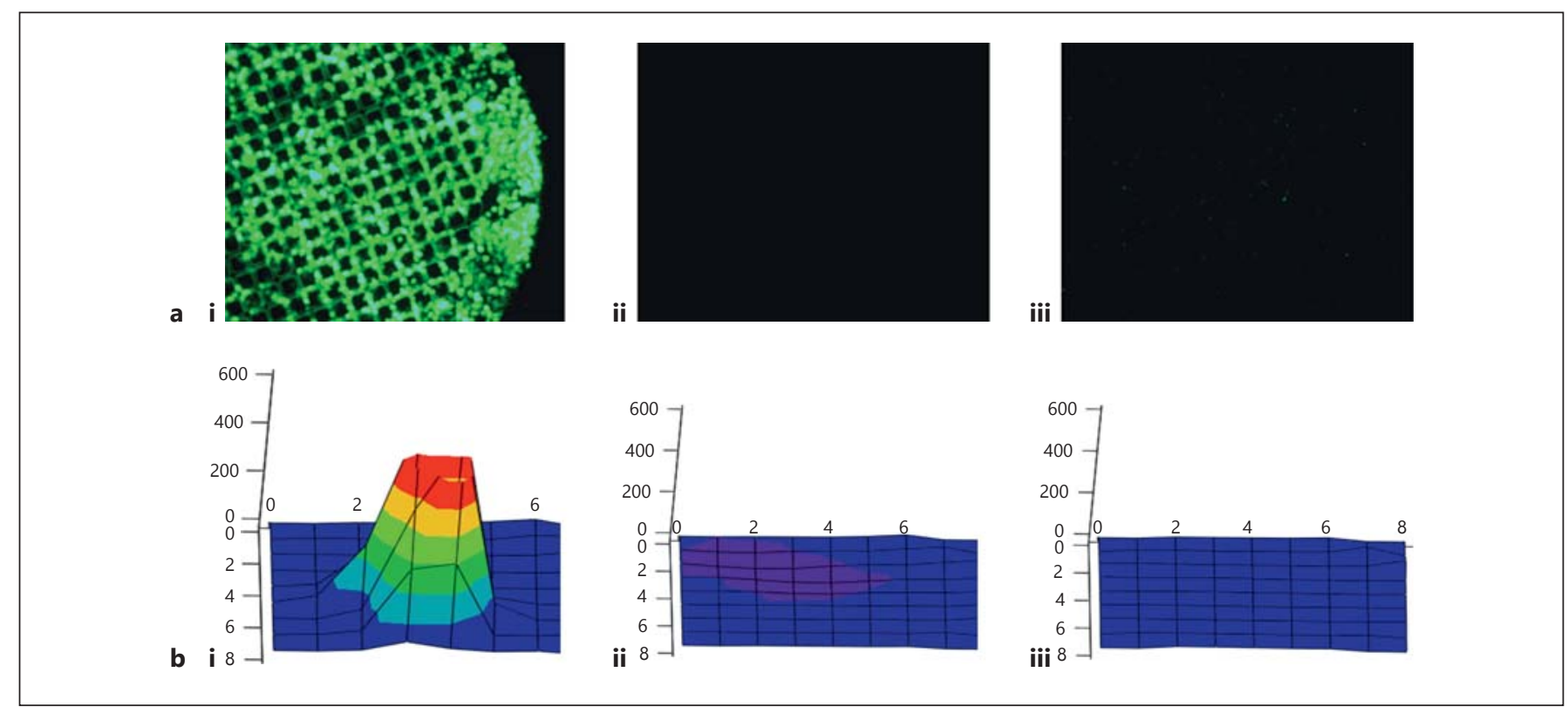

Fig. 1. a Representative fluorescence photomicrographs of NSCLC cells (A549) treated with $1.5 \times 10^{8}$ particles of Cy3AdGFP either immobilized on stainless steel mesh disks via anti-knob antibody (i), or mock-immobilized to control meshes formulated without anti-knob antibody (ii), or $1.5 \times 10^{8}$ particles of free-soluble Cy3AdGFP (iii). Original magnification $\times 40$. $\mathbf{b}$ Three-dimensional reconstruction (MathCad software) of GFP expression profiles

within $30 \mathrm{~min}$ from surgical excision. Ad.LacZ-tethered stents were deployed into the explanted bronchi and then were subsequently incubated for $24 \mathrm{~h}$ at $37^{\circ} \mathrm{C}$. Stented bronchi were removed from the media, washed with PBS, fixed and stained with $\beta$-gal. After staining, a longitudinal incision along the explanted bronchus was made to remove the stent and visualize the bronchial epithelium.

\section{Statistical Analysis}

Tumor volumes are expressed as the mean tumor volume \pm SEM. We analyzed differences using the Student t test. KaplanMeier survival was calculated from the date of tumor implant. The Mantel-Cox log-rank test was used to compare survival curves. A $p$ value $<0.05$ was considered to represent statistical significance for all tests.

\section{Results}

\section{In vitro Transduction of Lung Cancer Cells from}

Viral-Coated Metallic Mesh

Palliative relief of obstruction in the tracheobronchial tree, caused by either endobronchial tumor or extrinsic compression by parabronchial tumors, is the most common reason for deploying endobronchial stents. Even in the clinical setting of malignant extrinsic airway obstruc- in the wells treated with Ad-iNOS immobilized on stainless steel mesh disks via anti-knob antibody (i), mock-immobilized to control meshes (ii), or with equal amount of free-soluble Cy3AdGFP (iii). Z-axis values correspond to GFP fluorescence data (excitation $485 \mathrm{~nm} /$ emission $515 \mathrm{~nm}$ ) assessed separately in 68 individually scanned areas per well.

tion, there is frequently endoluminal extension of the neoplastic process. As a result, tracheobronchial stents often come into direct contact with tumor.

In order to determine if adenoviral gene delivery to airway epithelium could be achieved with stents, we developed an in vitro model system using small metallic mesh disks as a flat equivalent to endoluminal stents. Utilizing the anti-knob monoclonal antibody-tethering technology, but with bare metal attachment using PAA$\mathrm{BP}$ rather than via coating, we were able to achieve uniform and abundant tethering of the Cy3-labeled adenoviral vector to the metal struts of the mesh disks (data not shown). Using this system, we initially aimed to determine whether Ad.GFP-tethered mesh disks could transduce NSCLC cell lines in vitro. The transduction was assessed by fluorescence microscopy and quantified by live cell fluorimetry. The human NSCLC cell line A549 was successfully transduced in vitro via mesh-mediated delivery of Ad.GFP (fig. 1a, b). Cells in direct contact with the metal disk struts were most effectively transduced. In contrast, there was minimal transduction of the cells that were not in direct contact with the metallic struts of the disk. This spatial pattern of transduction reflects the 

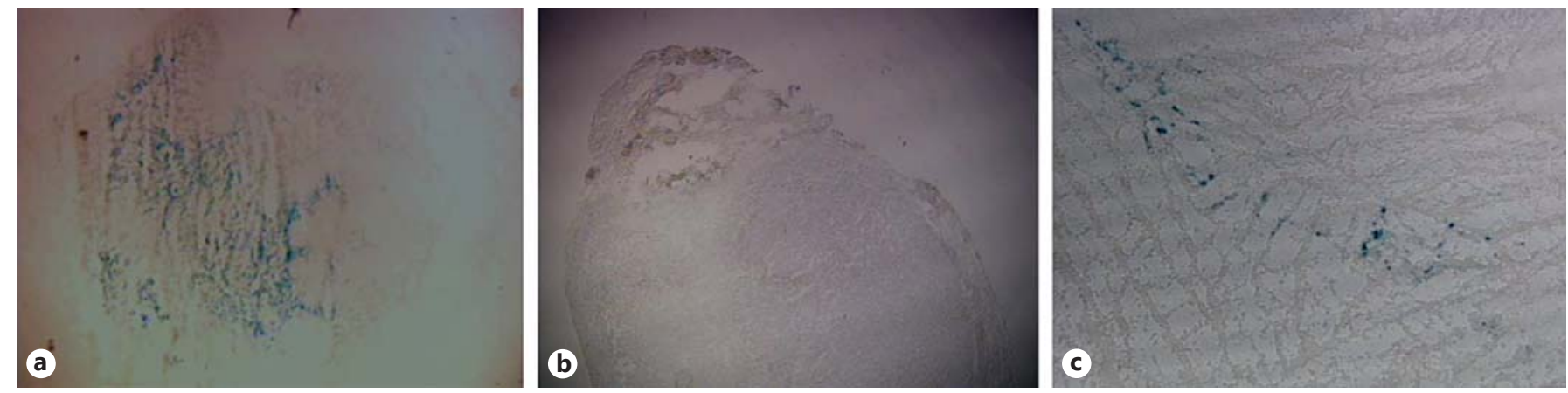

Fig. 2. Representative sections of flank tumor stained with $\beta$-gal. Flank tumors were treated with either mesh disks alone (a) or mesh disks immobilized with Ad.LacZ (b) that were surgically im- planted immediately adjacent to established L1C2 flank tumors. Alternatively, soluble Ad.LacZ was directly injected IT (c) into similar tumors. tight immobilization of mesh-associated vector with virtually no diffusion of transgene across the cell culture. Essentially no GFP expression was observed when the mesh disks were not coupled with anti-knob antibody prior to incubation with adenoviral vector (control mesh), excluding a nonspecific interaction between adenoviruses and disks as the significant mechanism responsible for vector immobilization. Free-soluble Ad. GFP was administered to the cell culture in the amount equivalent to the mesh-tethered vector. This yielded 8 -fold lower GFP expression levels $(\mathrm{p}<0.01)$. Moreover, since GFP expression from free-soluble Ad.GFP was uniformly distributed throughout the wells, the apparent 8.5-fold increase of total GFP expression underestimates the localized transduction efficacy of immobilized vector. Next, we individually scanned and interrogated each section of the well, specifically the highest expressing regions. We observed 67-fold higher transgene levels in cells treated by the mesh-tethered adenoviral vectors (fig. 1b). Qualitatively similar results were obtained for the murine NSCLC cell line L1C2 (data not shown).

\section{Transduction of Established NSCLC Tumors with}

\section{Mesh-Mediated Gene Delivery}

Having demonstrated successful in vitro transduction of NSCLC cell lines, we proceeded to determine if meshmediated gene delivery could be achieved in established tumors. Mesh disks tethered with Ad.LacZ were surgically implanted adjacent to L1C2 tumors, approximately $250 \mathrm{~mm}^{3}$, grown on the flank of immunocompetent Balb/C mice $(n=5)$. Transduction was allowed to occur over $48 \mathrm{~h}$, at which point the mice were sacrificed and tumors harvested. Sectioned tumors demonstrated significant $\beta$-gal staining, indicating that established tumors could be effectively transduced with this technique, and appeared superior to intratumoral Ad.LacZ injection (fig. 2a, c). Tumor sections obtained at sites distant from the mesh disk did not demonstrate $\beta$-gal staining (data not shown). Furthermore, no $\beta$-gal staining was observed with untethered control mesh disks (fig. 2b).

Murine flank tumors are a common model system to study the effects of various therapeutic interventions on tumor growth. The standard method of therapeutic vector administration is via direct intratumoral injection. In order to compare mesh disk-mediated gene delivery to the standard delivery, we injected flank tumors with Ad.LacZ in the usual manner. Tumor cells were successfully transduced with direct intratumoral injection (fig. 2c). Interestingly, upon sectioning, $\beta$-gal staining was present in a linear pattern throughout the tumor, likely representing the deposition of virus along the needle tract with subsequent transduction of surrounding cells. Although difficult to quantitate, mesh disk-mediated gene delivery appears to be at least as effective as direct intratumoral injection given the similar amount of $\beta$-gal staining using both delivery methods.

\section{A Model for Mesh-Mediated Delivery of Therapeutic} Genes to Established Flank Tumors

Previously, we have shown direct intratumoral injection of Ad.INF- $\beta$ to be effective in slowing the growth of established flank tumors [17]. To assess whether therapeutic genes could be delivered to established flank tumors using this methodology, Ad.INF- $\beta$ was coupled directly to metallic mesh disks. Disks coupled with Ad. INF- $\beta$, or Ad.LacZ as a control virus, were surgically implanted adjacent to established flank tumors $(n=5$ in each group). Once the tumors reached $125-150 \mathrm{~mm}^{3}$, vi- 
rus-impregnated mesh disks were implanted into the flanks alongside the tumors. In this pilot experiment, mesh disks coupled with Ad.INF- $\beta$ appeared to slow tumor growth over time as compared to control mesh disks (fig. 3a, b).

Within 12 days following mesh implantation, mean tumor volume was markedly decreased in the Ad.INF- $\beta$ mesh group compared to the Ad.LacZ-mesh group (290 vs. $636 \mathrm{~mm}^{3}, \mathrm{p}=0.11$ ), although the difference did not reach statistical significance. The growth of untreated tumors was equivalent to tumors treated with control Ad.LacZ-coupled mesh disks (data not shown).

\section{A Rat Model for Tracheobronchial Stent Placement and Maintenance}

In an attempt to approximate actual clinical scenarios, we next evaluated epithelial gene transfer using metallic stents deployed endoluminally in the tracheobronchial tree of live animals. We developed a model in which 1.5$\mathrm{mm}$ bare metal stents designed for insertion into human coronary arteries (Boston Scientific Corporation, Natick, Mass., USA) were deployed into rat tracheas via a small transverse airway incision. Using this procedure, periprocedural deaths were uncommon and most often resulted from tracheal rupture due to improper stent deployment. With proper positioning, the majority of rats $(n=14$, $>90 \%$ survival) remained alive for $>15$ days. There was no observed evidence of respiratory distress, stridor, wheezing or tachypnea in the postprocedure period. One rat died of unknown causes 3 days after stent placement.

Transduction of Tracheal Epithelium and Submucosal Glands Using Stent-Mediated Delivery of Ad.LacZ

Endovascular stents coupled with Ad.LacZ were deployed into the mid-trachea of Fisher 344 rats. Fortyeight hours after stent deployment, the rats were sacrificed and their tracheas harvested (fig. 4) and stained with $\beta$-gal. Staining with $\beta$-gal was readily apparent on the rat trachea, indicative of successful transduction of the tracheal epithelium by the adenoviral vector. On the epithelial surface, $\beta$-gal staining was most prominent in linear streaks that most likely represent the areas where the metal struts were in close contact with the trachea (fig. $4 \mathrm{~b}$ ). In contrast, there was no apparent $\beta$-gal staining when control stents, which lacked anti-knob antibody and thus were unable to couple Ad.LacZ, were utilized (fig. 4c, d). Similarly, unstented tracheas and tracheas stented with bare metal stents did not demonstrate $\beta$-gal staining (data not shown). Microscopic examination of the stained and sectioned trachea demonstrated superficial, heteroge-

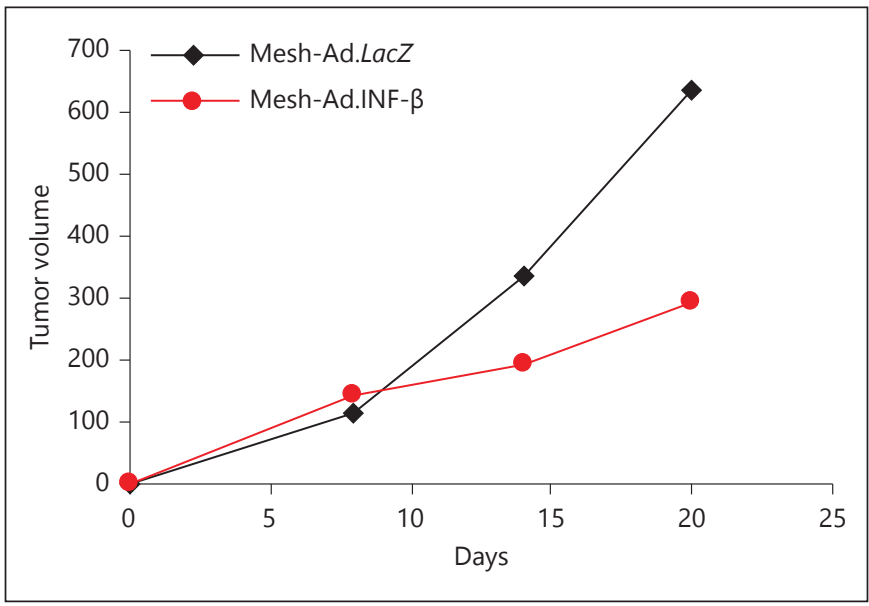

Fig. 3. L1C2 flank tumors were established in syngeneic Balb/C mice ( $n=5$ per treatment arm). Tumors were allowed to grow untreated until the mean tumor volume reached $100-125 \mathrm{~mm}^{3}$. Mesh disks, tethered to either Ad.LacZ control virus, or to Ad.INF- $\beta$ therapeutic virus $(\sim 2 \times 10 \mathrm{e} 7$ viral particles of each vector $)$, were surgically implanted immediately adjacent to the tumor. The tumor volume was followed serially with time.

neous staining of the tracheal epithelium in each section observed (fig. 5a).

Similar to the pattern observed with the mesh disks, areas between the metal struts of the stent did not appear to be transduced, as there was no $\beta$-gal staining present in these areas. Interestingly, in some sections, tissue transduction appeared to penetrate beyond the tracheal epithelium as demonstrated by submucosal gland staining (fig. 5b, c). Although only a few submucosal glands stained intensely with $\beta$-gal, approximately $25-50 \%$ of submucosal glands had some detectable $\beta$-gal staining, indicating a certain degree of successful subepithelial transduction.

\section{Ex vivo Transduction of Human Bronchi Using}

Stent-Mediated Delivery of Ad.LacZ

Ultimately, the clinical application of stent-mediated gene delivery technology in pulmonary diseases would necessitate the delivery of therapeutic vectors to the human tracheobronchial tree. Therefore, we postulated that human bronchial epithelium could be transduced ex vivo using this technique. Fifth-to-seventh generation human bronchi, with diameters of approximately $6-10 \mathrm{~mm}$, were isolated from explanted human lung specimens obtained at the time of lung transplantation under an IRB-approved protocol for assessment of explanted tissues. Ad.LacZ-coupled stents were deployed into the explanted 


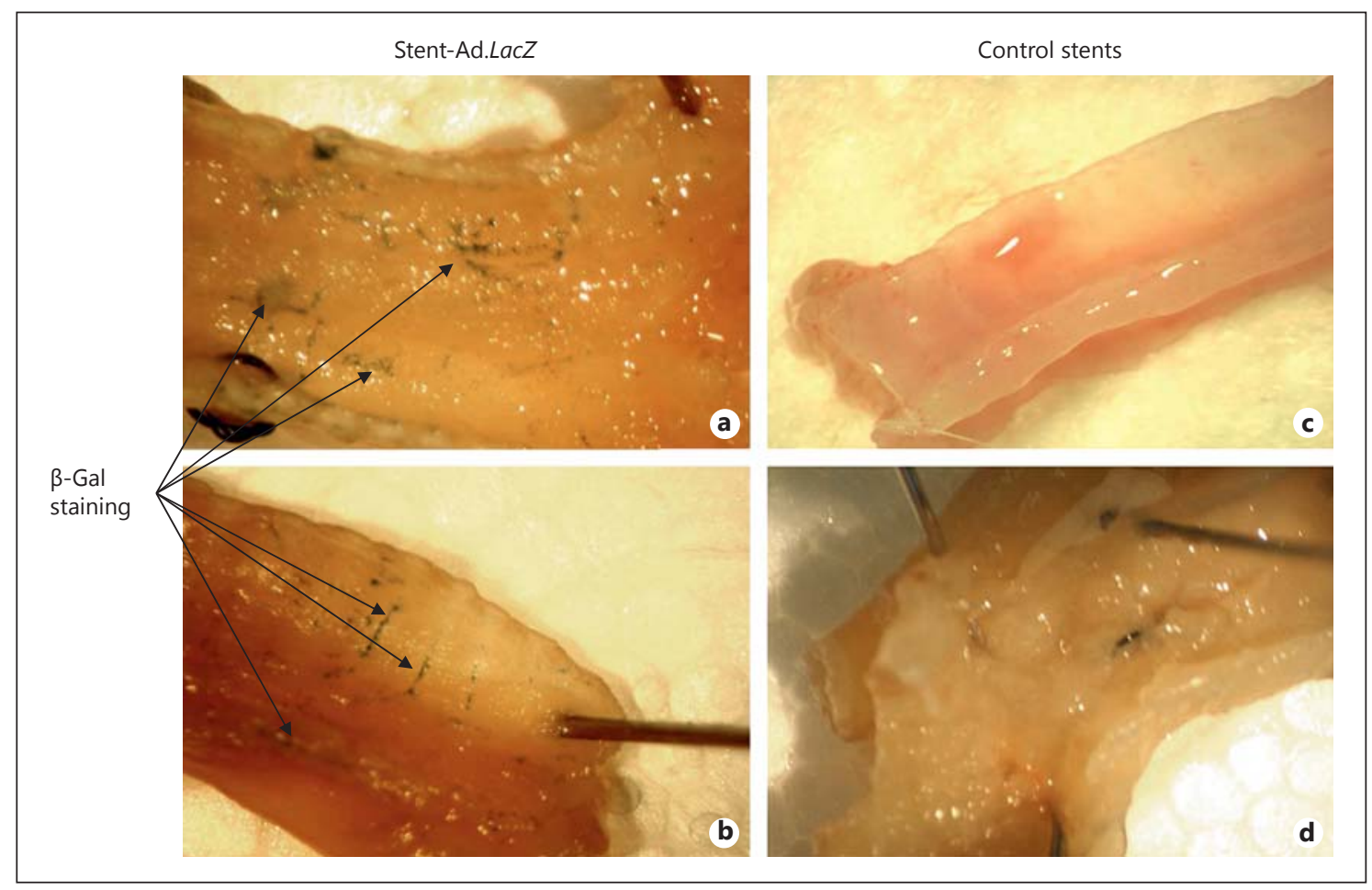

Fig. 4. Stents immobilized with Ad.LacZ (a, b) and noncomplexed stents $(\mathbf{c}, \mathbf{d})$ were deployed into rat tracheas and allowed to remain in place for $48 \mathrm{~h}$. Harvested tracheas, stained with $\beta$-gal substrate, were opened longitudinally (b). Streaks of $\beta$-gal activity corre-

bronchi and then these bronchial explants were placed directly into cell culture medium. After $24 \mathrm{~h}$, the stented bronchi were fixed and stained to assess for transduction. Similar to our findings in the rat trachea, visible linear $\beta$-gal staining was clearly evident on the bronchial epithelium (data not shown). Additionally, a fine transparent biofilm that coated the stent after removal also showed speckled $\beta$-gal staining.

\section{Development of an Endotracheal Rat Tumor Model}

We wished to develop a syngeneic intratracheal tumor model in immunocompetent rats such that we could begin to assess the efficacy of stent-mediated gene delivery. II-45 tumor cells, a mesothelioma cell line [17], were introduced intratracheally into immunocompetent Fisher 344 rats in order to develop endotracheal tumors (fig. 6a, b). The majority of rats $(\mathrm{n}=12,>90 \%)$ died between 7 and 12 days following tumor instillation. Necropsy revealed endotracheal tumors with tracheal obstruction as the putative cause of death. Study rats rarely demonstrated long-term survival $(<10 \%$ with $>20$ day survival) without evidence of endotracheal tumor formation. sponding to Ad.LacZ expression are seen in $\mathbf{a}$ and $\mathbf{b}$ in patterns consistent with the contact of stent struts to the tracheal mucosa. No $\beta$-gal activity was noted in sections of trachea in contact with noncomplexed stents.

\section{In vivo Model of Endobronchial Stent-Mediated}

Therapeutic Gene Delivery in Tumor-Bearing Rats

We then conducted a small pilot experiment in Fisher 344 rats utilizing the syngeneic intratracheal II-45 tumor model. Four days following the intratracheal tumor cell injection, we deployed either control Ad.LacZ-tethered stents $(n=5)$ or stents tethered to Ad.ratIFN- $\beta(n=5)$. Rats were followed for survival in comparison with rats with untreated (no stent placed) intratracheal tumors $(n=5)$. Placement of control stents resulted in a small improvement in survival. There was a further trend towards increased survival in rats treated with stents coupled with therapeutic Ad.INF- $\beta$ (fig. 7), although this improvement in survival was not statistically significant (data not shown).

\section{Discussion}

Over the last several decades, a series of investigations have been conducted with the goal of advancing treatment of various benign and malignant respiratory disorders with endobronchial gene transfer $[5,6]$. In summary, 

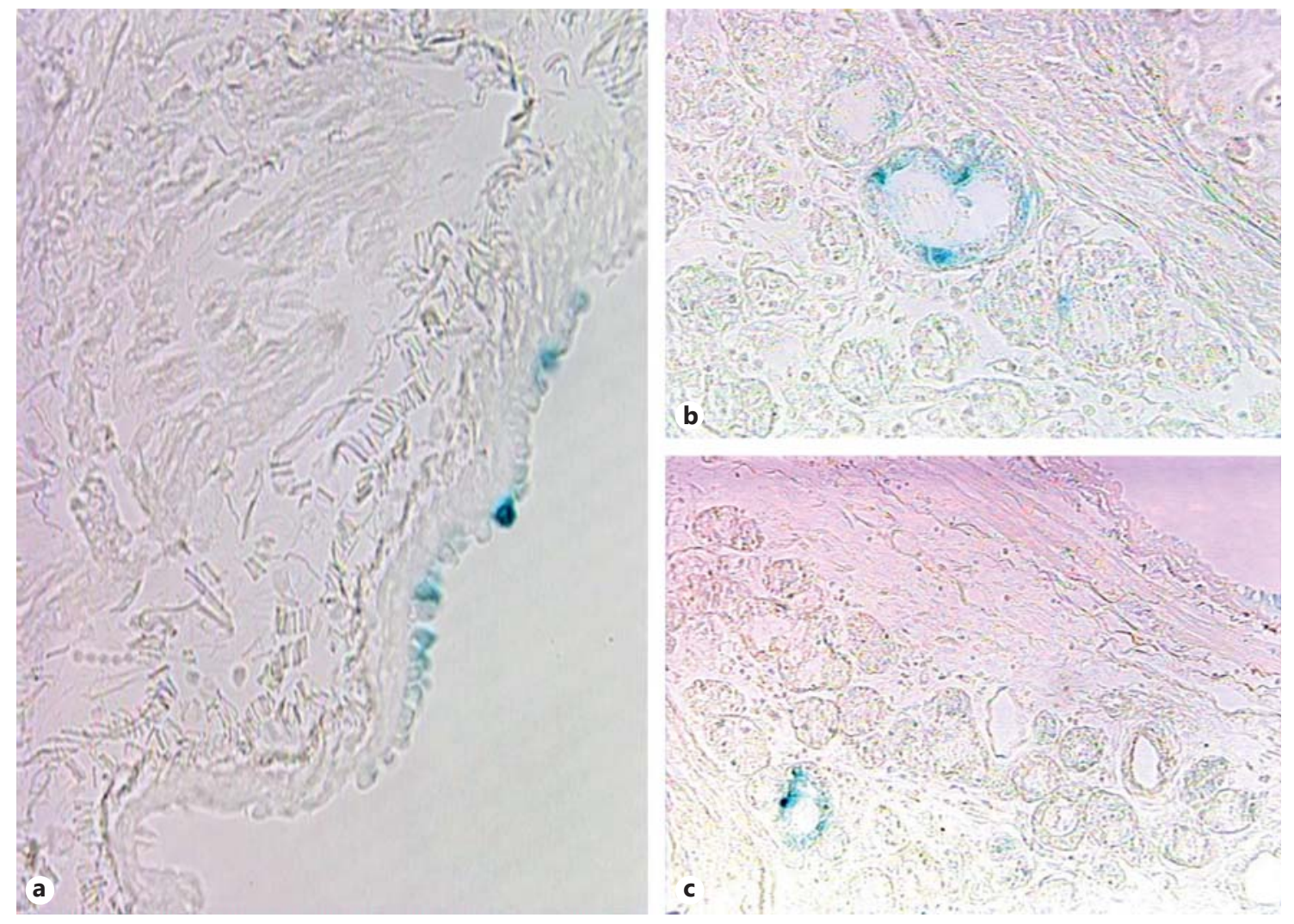

Fig. 5. The $\beta$-gal substrate-stained rat tracheas depicted in figure 4 were flash frozen and sectioned for light microscopy. Microscopic examination demonstrated superficial, heterogeneous staining of the tracheal epithelium (a); in some sections $(\mathbf{b}, \mathbf{c})$, tissue transduction appeared to penetrate beyond the tracheal epithelium into subjacent submucosal glands.

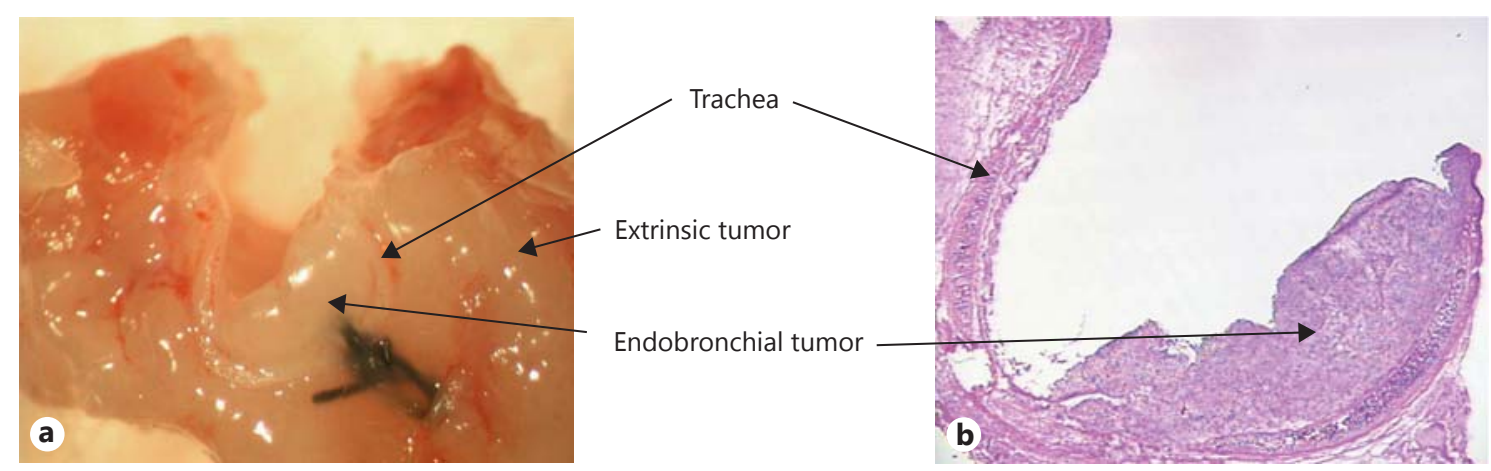

Fig. 6. Endotracheal II-45 tumors were established into rat tracheas and allowed to grow for 5 days before harvest. a A dissected photograph of gross rat trachea with endotracheal II-45 tumor, partially obstructing the airway lumen. Suture at the insertion site is visible in the lower right portion of the image. $\mathbf{b}$ Endotracheal tumors were then flash frozen and stained with HE. these studies have demonstrated reasonable degrees of safety and some degree of evidence of target cell transgene expression, but with demonstrable intrapulmonary inflammatory responses, and minimal overall clinical benefits [9-13, 18-22]. The latter likely reflects inefficient gene delivery and target cell transduction of in the tracheobronchial tree. It is evident that the natural protective mechanisms of mucociliary clearance and mucin production in the tracheobronchial tree provide obstacles to the delivery of desired therapeutic vectors. Conse- 


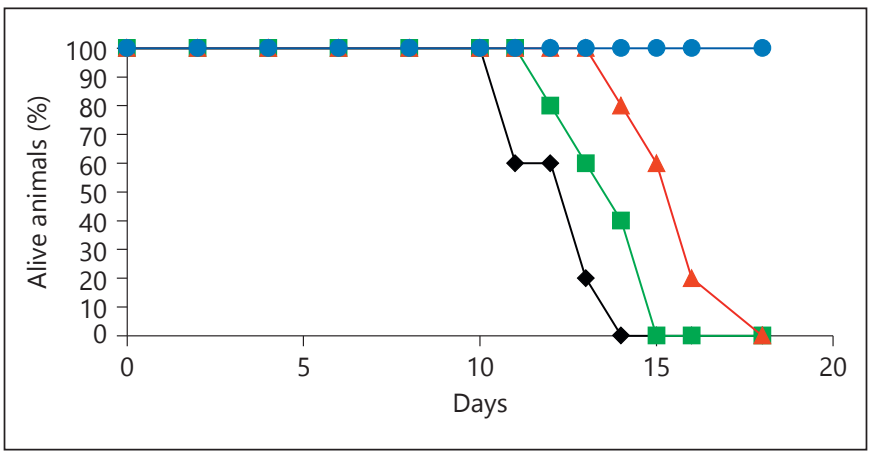

Fig. 7. Rats with endotracheal tumors were either treated with stents tethered to Ad.LacZ control virus (green line) or Ad.INF- $\beta$ therapeutic virus (red line). Control rats without endotracheal tumors were treated with noncomplexed bare metal stents (blue line). An additional group of rats receiving intratracheal II-45 tumor but no stent had the shortest survival, with $100 \%$ of animals in this group dead by day 14 (black line). There was a trend towards longer survival in the stent-INF- $\beta$ cohort, but the numbers of animals were too small to determine statistical significance.

quently, the development of vectors or gene delivery technology that could overcome these barriers may improve the efficiency and broaden the application of therapeutic intrabronchial gene delivery.

We have demonstrated here the ability to use a novel tethering technique to attach adenoviral vectors to bare metal surfaces using either mesh disks or endovascular stents. The mesh disks were capable of effectively transducing NSCLC cell lines in vitro, particularly when compared to transduction achieved with soluble virus. In vivo deployment of adenoviral-tethered stents in rat tracheas resulted in significant reporter gene expression in not only the epithelium, but also in the submucosal glands as well. Finally, we observed this same pattern of epithelial staining in ex vivo normal human bronchi.

The superior transduction ability of stent-mediated gene delivery as compared to soluble delivery (at least in vitro) is likely multifactorial. Firstly is the prolonged physical contact of the metallic stent struts - coupled to therapeutic Ad vectors - with the target bronchial epithelial cells. This prolonged contact time increased the degree of in vitro viral transduction when compared to soluble vector, and would likely prove superior in the clinical setting to viral transduction resulting from bronchoscopic instillation and/or aerosol delivery. Secondly, immobilized adenovirus may prove less immunogenic in vivo, with decreased Ad vector accessibility to immune effector cells and/or neutralization by antiadenoviral antibodies. Thirdly, mechanical forces applied to the bronchial epi- thelium by the expanded metallic stent structure may alter the bronchial epithelium by disrupting tight junctions or altering cell adhesion molecules or adenoviral receptor expression, making the bronchial epithelium, and perhaps subepithelial cellular structures, more conducive to transduction. Finally, the classical paradigm of Ad cell entry implies involvement of 2 receptor systems: coxsackie adenovirus receptor (CAR) and integrin receptors. High-affinity CAR-knob interaction serves to dock adenovirus near the cell surface to provide an internalization event triggered by the low-affinity interaction between $\alpha_{v} \beta_{3 / 5}$ integrins and penton base of Ad. To this end, viral immobilization by approximating the vector to target cells obviates the dependence of transduction on apical CAR and $\alpha_{\mathrm{v}} \beta_{1}$ integrin expression in normal and malignant bronchial epithelia [23].

Although we demonstrated stent-mediated marker gene expression in the in vivo rat tracheal model and the ex vivo human bronchus model, the amount of marker gene expression in the bronchial epithelium was relatively low, particularly compared with the data from the in vitro experiments with mesh disks. There were several limitations to this technique, including poor apposition between the stents and the bronchial epithelium given the fact that the coronary stents were neither designed nor sized for insertion in the tracheobronchial epithelium in this ex vivo experiment. One would ultimately expect better tissue contact, and thereby improved transgene expression, with appropriately sized tracheobronchial stents tethered to marker and/or therapeutic genes. An additional limitation was the questionable ex vivo survival of epithelial cells in explanted human bronchi. Apoptotic epithelial cell death postexplantation would certainly result in decreased efficiency of adenoviral-mediated transgene expression, regardless of the mode of transgene delivery. Finally, the GFP reporter utilized in our in vitro experiments is at least an order of magnitude more sensitive than the LacZ reporter employed in vivo. Therefore, the extent of transduction in the flank tumors as well as in tracheal and bronchial tissue may be underestimated in our in vivo experiments.

Of particular interest is the ability of stent-mediated gene delivery to transduce submucosal glands. Transduction of submucosal glands using aerosolized delivery of vectors has proven to be inefficient. Effective transduction of the submucosal glands may be important for successful treatment of airway disorders such as CF. Since we utilized replication-incompetent adenoviral vectors, bronchial epithelium injury and/or temporary denudation at the site of the stent placement must have permitted 
adenoviral access to the submucosal glands. Alternatively, the physical presence of the metallic stent abutting the bronchial epithelium with resultant distortion and disruption of apical tight junctions between epithelial cells may have contributed to subepithelial transduction.

An extremely important attribute of the described stent-mediated gene delivery system is the tight spatial control of the gene expression observed both in vitro and in vivo. Although less stringent vector adherence allowing for local spread of recombinant vector might prove beneficial, contrastingly adenoviral stent conjugation may offer major advances from a safety standpoint for intrapulmonary gene delivery. Both aerosolized delivery and direct bronchoscopic instillation result in significant distal airway and alveolar vector deposition. This diffuse, peripheral distribution of vector carries the potential for inducing severe inflammatory responses, possibly resulting in acute lung injury [18].

\section{Other Indications for Stent-Mediated Gene Transfer} in the Tracheobronchial Tree

Unfortunately, the deployment of endobronchial stents is limited at the current time to the central airways (trachea, main stem bronchi, bronchus intermedius, some lobar bronchi), therefore limiting the value of this delivery method in diffuse pulmonary disease such as CF, a1-antitrypsin deficiency, COPD or asthma. There may, however, prove to be a benefit of achieving high levels of wild-type (wt) CFTR expression in the central airways of patients with CF, specifically in terms of alteration of the disease phenotype, even in the absence of more diffuse tracheobronchial expression of wtCFTR.

Stent-mediated gene delivery could prove beneficial in the treatment of aerodigestive tract fistulae, tracheobronchial malacia, or even to decrease the risk of rejection in lung transplantation. The most likely future indication for stent-mediated gene delivery, however, would be for treatment of localized abnormalities of the large airways, such as seen with obstructing NSCLC lesions. Patients with these lesions would putatively derive dual benefit from stent-mediated gene delivery - palliative improvement in airway patency with stent deployment in addition to the antitumor effects of high-level local expression of therapeutic genes, altering the host antitumor immune response, replacing deleted or mutated tumor suppressor genes, or inhibiting overexpressed genes.

One major downside of this approach to transduction of the tracheobronchial epithelium would be the presence of a metallic foreign body in the central airways of patients with potentially marginal underlying lung func- tion, with all the attendant stent-related complications well documented in patients undergoing self-expandable metallic stent placement for benign or malignant airway disease. These potential complications include stent migration, fracture, airway perforation, mucoid impaction and, most seriously, the development of obstructing granulation tissue. For these reasons, stent-mediated gene delivery would likely initially be limited to patients with advanced lung cancer, who would otherwise have a limited lifespan in which to experience stent-related complications. Other options would be to conjugate adenoviral (or other vector types) to silicone or composite stents that would be readily removable at short time intervals after insertion, allowing for an appropriate time period for maximal transduction of the underlying epithelium. Ultimately, bioabsorbable stents would prove ideal for stable and high-level gene delivery to the tracheobronchial mucosa [24-26].

The tremendous potential of stent-mediated (or mesh disk-mediated) delivery of therapeutic genes to tumors is illustrated by the murine flank and rat tracheal in vivo experiments described above. Mesh-mediated intratumoral delivery of the murine IFN- $\beta$ gene carried by the conjugated recombinant Ad vector significantly enhanced antitumor efficacy compared to direct intratumoral (IT) injection of Ad.muIFN- $\beta$. The limitations of IT gene delivery in clinical trials, including the need for repeated injection and risk of systemic dissemination of vector, may be obviated by clinical implementation of IT implantation of gene-coated disks, with advantages similar to carmustine wafers for peritumoral chemotherapy delivery in brain tumors [27].

One additional concern regarding the use of viral vectors, and adenoviruses in particular, for intrapulmonary gene delivery is the documented association of these constructs with humoral and cellular immune responses, as well as cytokine release syndrome, when utilized in a variety of human clinical trials. In fact, our group has a significant clinical experience with intrathoracic delivery of recombinant adenoviruses in a series of phase I and phase II clinical trials for mesothelioma and malignant pleural effusions dating back to $1995[5,6]$. We have routinely seen fevers starting within 4-6 h of intrapleural infusion of adenoviral vector, persisting in an intermittent fashion over the ensuing $12-36 \mathrm{~h}$. These are generally well tolerated, palliated with antipyretics, and rarely accompanied by more serious aspects of the cytokine release syndrome, such as hypotension and hypoxemia.

Bronchoscopic delivery of recombinant adenoviruses, primarily in phase I clinical trials of delivery of the 
wtCFTR gene in patients with $\mathrm{CF}$, has been associated with inflammatory pneumonitis and hypoxia [18-20]. This has been theorized to be secondary to passage of the adenoviral vector into the alveolar compartment of the lung parenchyma, where interaction of the viral vector with intra-alveolar macrophages is the major factor in the induction of the inflammatory response. It is unlikely that stent-mediated adenoviral gene delivery to the epithelium of the central airways (trachea/main stem bronchi) would result in sufficient leakage of vector into the lung parenchyma to cause an inflammatory response. There may be some localized airway inflammation secondary to humoral and cellular responses to the adenoviral vector complexed to the metallic stent, but this would be unlikely to cause airway obstruction given the presence of the stent to maintain patency. Our group does have experience with the use of nonviral vectors for stent-mediated gene delivery in cell culture [28], and these may be worthwhile studying in our preclinical rat model of syngeneic intratracheal tumor in the future.

The syngeneic rat tracheal tumor experiment, albeit highly preliminary, and with few animals in each treatment group, is highly provocative in that it suggests that stents complexed with therapeutic genes can improve survival compared with stent insertion alone. Airway stenting using control stents seemingly had a limited effect on survival. At necropsy we found endotracheal tumor occluding the trachea adjacent to both the proximal and distal ends of the stent. Additionally, we expect tumor growth between the struts of these uncovered stents. It is therefore possible that covered stents and stents that completely span the length of endotracheal tumor and/or more adequately fit target airway dimensions with circumferential mucosal apposition might have a more significant effect on rat survival. With this model we could more effectively assess the effects of stent-delivered gene therapy.

In theory, a patient with advanced NSCLC involving the central airway, but who also had distant metastases, could undergo stent-mediated delivery of IFN- $\beta$ (or other immunostimulatory genes) and have palliative improvement in central airway patency, reduction in local tumor burden, as well as responses at distant tumor sites. In combination with current chemotherapy and radiation therapy, the ability to deliver therapeutic genes in a focal and targeted fashion may ultimately improve outcomes in this devastating disease.

\section{References}

1 Dubey S, Powell CA: Update in lung cancer 2006. Am J Respir Crit Care Med 2007;175: 868-874.

2 McKenna RJ Jr: Surgical management of primary lung cancer. Semin Oncol 2007;34:250255.

-3 Brahmer JR, Ettinger DS: Non-small cell lung cancer: adjuvant and neo-adjuvant chemotherapy. Respirology 2007;12:320-325.

-4 Okawara G, Mackay JA, Evans WK, Ung YC, Lung Cancer Disease Site Group of Cancer Care Ontario's Program in Evidence-Based Care: Management of unresected stage III non-small cell lung cancer: a systematic review. J Thorac Oncol 2006; 1:377-393.

-5 Vachani A, Moon E, Wakeam E, Albelda SM: Gene therapy for mesothelioma and lung cancer. Am J Respir Cell Mol Biol 2010;42:385393.

6 Vachani A, Moon E, Wakeam E, Haas AR, Sterman DH, Albelda SM: Gene therapy for lung neoplasms. Clin Chest Med 2011;32:865885.

7 Kruklitis R, Sterman DH: Endobronchial Gene Therapy. Semin Respir Crit Care Med 2004:25:433-442.

$\checkmark 8$ Beck SE, Jones LA, Chesnut K, Walsh SM, Reynolds TC, Carter BJ, Askin FB, Flotte TR, Guggino WB: Repeated delivery of adeno-associated virus vectors to the rabbit airway. J Virol 1999;73:9446-9455.
9 Keedy V, Wang W, Schiller J, Chada S, Slovis B, Coffee K, Worrell J, Thet LA, Johnson DH, Carbone DP: Phase I study of adenovirus p53 administered by bronchoalveolar lavage in patients with bronchioloalveolar cell lung carcinoma: ECOG 6597. J Clin Oncol 2008;26: 4166-4171.

10 Cipolla DC, Gonda I, Shak S, Kovesdi I, Crystal R, Sweeney TD: Coarse spray delivery to a localized region of the pulmonary airways for gene therapy. Hum Gene Ther 2000;11:361371.

11 Harvey BG, Leopold PL, Hackett NR, Grasso TM, Williams PM, et al: Airway epithelial CFTR mRNA expression in cystic fibrosis patients after repetitive administration of a recombinant adenovirus. J Clin Invest 1999; 104:1245-1255

12 Flotte TR, Laube BL: Gene therapy in cystic fibrosis. Chest 2001;120:124S-131S.

13 Pilewski JM: Gene therapy for airway diseases: continued progress toward identifying and overcoming barriers to efficiency. Am J Respir Cell Mol Biol 2002;27:117-121.

14 Fishbein I, Alferiev IS, Nyanguile O, Gaster R, Vohs JM, Wong GS, Felderman H, Chen IW, Choi H, Wilensky RL, Levy RJ: Bisphosphonate-mediated gene vector delivery from the metal surfaces of stents. Proc Natl Acad Sci USA 2006;103:159-164.
15 Odaka M, Sterman DH, Wiewrodt R, Zhang Y, Kiefer M, Amin KM, Gao GP, Wilson JM, Barsoum J, Kaiser LR, Albelda SM: Eradication of intraperitoneal and distant tumor by adenovirus-mediated interferon- $\beta$ gene therapy is attributable to induction of systemic immunity. Cancer Res 2001;61:62016212.

16 Odaka M, Wiewrodt R, DeLong P, Tanaka T, Zhang Y, Kaiser L, Albelda S: Analysis of the immunologic response generated by $\mathrm{Ad}$. IFN- $\beta$ during successful intraperitoneal tumor gene therapy. Mol Ther 2002;6:210-218.

17 Elshami AA, Kucharczuk JC, Zhang HB, Smythe WR, Hwang HC, Litzky LA, Kaiser LR, Albelda SM: Treatment of pleural mesothelioma in an immunocompetent rat model utilizing adenoviral transfer of the herpes simplex virus thymidine kinase gene. Hum Gene Ther 1996;7:141-148.

18 Crystal RG, McElvaney NG, Rosenfeld MA, Chu CS, Mastrangeli A, et al: Administration of an adenovirus containing the human CFTR cDNA to the respiratory tract of individuals with cystic fibrosis. Nat Genetics 1994;8:42-51.

19 Bellon G, Michel-Calemard L, Thouvenot D, Jagneaux V, Poitevin F, et al: Aerosol administration of a recombinant adenovirus expressing CFTR to cystic fibrosis patients: a phase I clinical trial. Hum Gene Ther 1997;8: $15-25$. 
20 Zuckerman JB, Robinson CB, McCoy KS, Shell R, Sferra TJ, et al: A phase I study of adenovirus-mediated transfer of the human cystic fibrosis transmembrane conductance regulator gene to a lung segment of individuals with cystic fibrosis. Hum Gene Ther 1999;10: 2973-2985.

21 Aitken ML, Moss RB, Waltz DA, Dovey ME, Tonelli MR, et al: A phase I study of aerosolized administration of tgAAVCF to cystic fibrosis subjects with mild lung disease. Hum Gene Ther 2001;12:1907-1916.

-22 Perricone MA, Morris JE, Pavelka K, Plog MS, O'Sullivan BP, et al: Aerosol and lobar administration of a recombinant adenovirus to individuals with cystic fibrosis. II. Transfection efficiency in airway epithelium. Hum Gene Ther 2001;12:1383-1394.
23 Davison E, Kirby I, Whitehouse J, Hart I, Marshall JF, Santis G: Adenovirus type 5 uptake by lung adenocarcinoma cells in culture correlates with Ad5 fibre binding is mediated by $\alpha_{v} \beta 1$ integrin and can be modulated by changes in $\beta 1$ integrin function. Gene Med 2001;3:550-559.

24 Korpela A, Aarnio P, Sariola H, Törmälä P, Harjula A: Bioabsorbable self-reinforced poly-L-lactide, metallic, and silicone stents in the management of experimental tracheal stenosis. Chest 1999;115:490-495.

25 Zhu GH, Ng AH, Venkatraman SS, Boey FY, Wee AL, Trasti SL, Yee Lim LH: A novel bioabsorbable drug-eluting tracheal stent. Laryngoscope 2011;121:2234-2239.
6 Chao YK, Liu KS, Wang YC, Huang YL, Liu SJ: Biodegradable cisplatin-eluting tracheal stent for malignant airway obstruction: in vivo and in vitro studies. Chest 2013;144:193-199.

27 Salvati M, D'elia A, Frati A, Brogna C, Santoro A, Delfini R: Safety and feasibility of the adjunct of local chemotherapy with biodegradable carmustine (BCNU) wafers to the standard multimodal approach to high grade gliomas at first diagnosis. J Neurosurg Sci 2011;55:1-6.

28 Ma G, Wang Y, Fishbein I, Yu M, Zhang L, Alferiev IS, Yang J, Song C, Levy RJ: Anchoring of self-assembled plasmid DNA/antiDNA antibody/cationic lipid micelles on bisphosphonate-modified stent for cardiovascular gene delivery. Int J Nanomedicine 2013;8:1029-1035. 


\section{Erratum}

The authors of the article entitled 'Stent-mediated gene delivery for site-specific transgene administration to the airway epithelium and management of tracheobronchial tumors' [Respiration 2014;88:406-417, DOI: 10.1159/000368164] wish to publish the following correction.

On page 406 the name of an author, Dr. Ivan S. Alferiev, is missing. The correct author list should be as follows:

Robert J. Kruklitis $^{c} \quad$ Ilia Fishbein $^{\mathrm{b}}$ Sunil Singhal ${ }^{\mathrm{a}}$ Veena Kapoor ${ }^{\mathrm{a}}$ Robert J. Levy ${ }^{\mathrm{b}}$ Ivan S. Alferiev ${ }^{\mathrm{b}}$ Daniel H. Sterman ${ }^{\mathrm{a}}$

aSection of Interventional Pulmonology and Thoracic Oncology, Pulmonary, Allergy and Critical Care Division, University of Pennsylvania Medical Center, and ${ }^{b}$ Division of Pediatric Cardiology, Children's Hospital of Philadelphia, Philadelphia, Pa., and 'Division of Pulmonary and Critical Care, Lehigh Valley Health Network, Allentown, Pa., USA 